\title{
Water Diffusion and Fracture Behavior in Nano- Porous Low-K Dielectric Film Stacks
}

\section{Citation}

$\mathrm{Li}$, Han, Ting T. Tsui, and Joost J. Vlassak. Forthcoming. Water diffusion and fracture behavior in nano-porous low-k dielectric film stacks. Journal of Applied Physics 105.

\section{Published Version}

http://jap.aip.org/

\section{Permanent link}

http://nrs.harvard.edu/urn-3:HUL.InstRepos:3121069

\section{Terms of Use}

This article was downloaded from Harvard University's DASH repository, and is made available under the terms and conditions applicable to Open Access Policy Articles, as set forth at http:// nrs.harvard.edu/urn-3:HUL.InstRepos:dash.current.terms-of-use\#OAP

\section{Share Your Story}

The Harvard community has made this article openly available.

Please share how this access benefits you. Submit a story.

Accessibility 


\title{
Water diffusion and fracture behavior in nano-porous low-k dielectric film stacks
}

\author{
Han Li ${ }^{1}$, Ting T.Tsui ${ }^{2}$, Joost J.Vlassak ${ }^{1 *}$ \\ ${ }^{1}$ School of Engineering and Applied Sciences, Harvard University, 29 Oxford Street, \\ Cambridge, Massachusetts 02138-2901, USA \\ ${ }^{2}$ Department of Chemical Engineering, Nanotechnology Institute, University of \\ Waterloo, 200 University Avenue West, Waterloo, Ontario, Canada N2L 3G1
}

\begin{abstract}
Among various low-dielectric constant ('low-k') materials under development, organosilicate glasses (OSG) containing nanometer-size pores are leading candidates for use as intra-metal dielectrics in future microelectronics technologies. In this paper, we investigate the direct impact of water diffusion on the fracture behavior of film stacks that contain porous OSG coatings. We demonstrate that exposure of the film stacks to water causes significant degradation of the interfacial adhesion energy, but that it has negligible effect on the cohesive fracture energy of the nanoporous OSG layer. Isotope tracer diffusion experiments combined with dynamic secondary ion mass spectroscopy (SIMS) show that water diffuses predominantly along the interfaces, and not through the porous films. This unexpected result is attributed to the hydrophilic character of the interfaces.
\end{abstract}

Keywords: Dielectrics, porous, water diffusion, cracking, SIMS

*Corresponding author. e-mail: vlassak@esag.harvard.edu 


\section{Introduction}

To boost signal transmission and reduce power dissipation, organosilicate glasses (OSG) have been introduced in high-performance circuits to replace silicon dioxide as intra-metal insulator materials. ${ }^{1,2}$ OSG materials have a silica-like backbone structure but with a fraction of the $\mathrm{Si}-\mathrm{O}$ bonds replaced by organic groups such as $-\mathrm{CH}_{3}$, to reduce the overall dielectric constant of the material..$^{3-5}$ The relative dielectric constant ( $k$ value) of dense OSG is limited to values greater than about $2.7 .^{6-8}$ It is expected that materials with even lower dielectric constants are needed for future generations of integrated circuits. To this end, a new class of OSG materials with $k$ values as low as 2.0 have been successfully synthesized using plasma-enhanced chemical vapor deposition (PECVD), and they are considered leading candidates for use as interconnection dielectrics in emerging technologies. ${ }^{6-9}$ The low dielectric constants of these materials are achieved by introducing nano-scale pores into the dense matrix of their predecessors.

Successful implementation of porous dielectrics in interconnect structures poses numerous challenges. Compared to their dense counter parts, porous dielectrics are expected to possess a much reduced cohesive strength and poorer adhesion with adjacent layers, and are more prone to the absorption of reactive chemicals during device fabrication. It has been reported that water can diffuse quite effectively into film stacks containing dielectrics layer, even though the dielectric materials are usually hydrophobic. ${ }^{10-13}$ The ingress of water into the dielectrics stacks negatively impacts both the electrical performance of the devices and their mechanical integrity. On the electrical side, water has a relative dielectric constant of approximately 80 owing to the polar $\mathrm{O}-\mathrm{H}$ bonds, so that even a small amount of water uptake can be fatal to the overall dielectric characteristics. Water also influences the leakage current of the dielectrics adversely. From the mechanical standpoint, the presence of water decreases the resistance of the dielectric materials to various forms of fracture caused by stress-corrosion (i.e., subcritical crack growth). ${ }^{12,14-16}$ Small cracks can grow over time even when the mechanical driving force is well below the material's intrinsic fracture resistance, causing reliability issues and yield loss.

Subcritical cracking in dielectric thin films has been studied extensively. With regards to subcritical delamination, two types of measurements have been reported depending on how the reactive species are transported to the crack tip. In one type of experiment, dry samples are tested in an atmosphere with a controlled water partial pressure, where reactive species are transported to the crack tip in-situ during the fracture process. Crack 
velocity, $v$, is then measured as a function of applied energy release rate, $G$. This approach has been traditionally taken to study stress-corrosion in bulk glasses, ${ }^{17,18}$ and it has been adopted for dielectric thin films as well. ${ }^{14-16,19}$ Alternatively, samples can be immersed in water prior to testing. The immersion time is controlled to allow a certain amount of diffusion to occur. Immediately after soaking, the sample is tested in an inert environment. As there is no transport of reactive species from the environment to the crack tip, only species already present in the sample as a result of diffusion are active in the subcritical fracture process. ${ }^{12,13}$ We term this approach the "soak-then-test" (STT) method. This method has been used to demonstrate that significant adhesion degradation can occur in dense OSG film stacks as a result of the ingress of reactive species such as water. By measuring the adhesion energy as a function of soaking time, the STT approach also allows determination of the water diffusivity in the film stacks. ${ }^{12,13}$

Nanoporous OSG capped with a SiCN diffusion barrier constitutes an important model system for future generations of microelectronic devices. ${ }^{20}$ To the best of our knowledge, an integrated study of this material system focusing on water diffusion and its impact on fracture is yet to be conducted. In this study, we first characterize the effect of water exposure on the fracture behavior of the OSG and the OSG/SiCN interface. Second, we probe the water diffusion path and concentration profile using an oxygen isotope and dynamic SIMS measurements. Xu et al. have used a similar approach to study water diffusion along the $\mathrm{TiN} / \mathrm{SiO}_{2}$ interface. ${ }^{21}$ Interpreting the result from fracture and SIMS measurements allows us to identify the main diffusion path, and extract the diffusion coefficient of water along that path. The implications of our study for the fracture properties of dielectric structures in the presence of water are discussed. 


\section{Experimental}

\section{A. Materials and sample fabrication}

Porous OSG films were deposited onto 300-mm (100)-Si wafers by means of plasmaenhanced chemical vapor deposition (PECVD) using diethoxymethylsilane (DEMS) as a precursor. The depositions were performed at a temperature of $250^{\circ} \mathrm{C}$. Helium and oxygen gas were used as carrier gas and oxidizer, respectively. A proprietary aromatic porogen was added to the gas mixture during deposition to form the sacrificial porogen phase. Pores were formed in the films after the deposition process by removing the volatile porogen phase from the as-deposited films. This operation was performed by irradiating the as-deposited films at $400{ }^{\circ} \mathrm{C}$ in vacuum with broadband $\mathrm{UV}$ radiation for a length of time that depended on both film thickness and porogen loading. The porous films were subsequently capped with a $300 \mathrm{~nm}$ SiCN coating using PECVD. OSG films with three different levels of porosity were deposited. Details about the film stacks used in this study are summarized in Table 1. For the low-k dielectric with intermediate porosity, we also modified either the OSG/SiCN or the OSG/Si interface. Specifically for film stack ULK-1-pt, the surface of the OSG layer was treated with a helium plasma for 30 seconds immediately prior to the deposition of the SiCN. For the ULK-1-liner stack, a silicon dioxide liner layer of approximately $5-10 \mathrm{~nm}$ was deposited on the silicon surface prior to OSG deposition, to reduce fast water diffusion along the Si/OSG interface. The relative dielectric constant of the coatings was measured using a Quantox XP metrology system (KLA-tencor Corp., Milpitas, California). Film thicknesses were measured using spectroscopic ellipsometry (Woollam VASE spectrometer, Lincoln, NE). The silicon substrates had a thickness of $780 \pm 10 \mu \mathrm{m}$, and were polished on both sides.

The cohesive fracture energy of the OSG films was measured using the doublecantilever beam (DCB) method. The DCB specimens were fabricated using the following procedure. First the film stacks were sandwiched between two pieces of silicon using a 5$\mu \mathrm{m}$ thick spin-on epoxy (Epotek_353ND). Prior to spin-coating the epoxy, a 40-nm Ti adhesion layer and a 400-nm Cu layer were sputter deposited onto the OSG film stack to increase the distance between the OSG and SiCN films and the epoxy and to minimize any energy dissipation in the epoxy during the measurement. The epoxy was cured at $120^{\circ} \mathrm{C}$ for 1 hour. The resulting sandwich was diced into six specimens with dimensions of $60 \mathrm{~mm} \times 6.8 \mathrm{~mm}$ and with the long edge parallel to the $<110>$ direction of the Si wafer. The dicing was performed using a Disco ADA-321 high-speed saw. Pre-cracks were made at one end of the DCB specimens by driving a razor blade between the two silicon 
substrates until a crack with a length of approximately $10 \mathrm{~mm}$ was created.

The adhesion energy of the OSG/SiCN interfaces was measured using the four-point bend (4-PB) technique. Fabrication of the 4-PB specimens is similar to that for the DCB specimen, except the creation of the pre-crack: The pre-crack in the 4-PB specimen was made by machining a notch in one of the Si substrates using a high speed-dicing saw (Disco ADA-321). Cracks initiate at the notch corners upon loading, and penetrate into the OSG/SiCN interface. The dimensions of the 4-PB specimens were the same as for the DCB specimens.

All DCB and 4-PB specimens were baked at $190^{\circ} \mathrm{C}$ for 3 hours using a hotplate under flowing nitrogen gas, and stored in desiccators before the fracture measurements. At least five specimens were prepared for each condition.

\section{B. Methods}

We used the STT approach to measure the variation of the cohesive fracture energy of the OSG and the adhesion energy of the OSG/SiCN interface as a function of water submersion time. The baked samples were submerged in distilled water at $22-23^{\circ} \mathrm{C}$ for periods of time ranging from less than a second to 32 hours. After soaking, the samples were blow dried with nitrogen gas and immediately tested inside an environmental cell. Relative humidity in the environmental cell was kept below $11 \%$ by flowing high purity nitrogen gas.

In the DCB test, the specimens were loaded by separating the Si substrates on the side of the pre-crack at a constant speed of $0.5 \mu \mathrm{m} / \mathrm{s}$, resulting in a crack growth rate of approximately $100 \mu \mathrm{m} / \mathrm{s}$. During the experiment, the load $P$ was measured using a precision load cell (Sensotec-31, Honeywell Sensotec) with a resolution of a few $\mu N$. The energy release rate for the double-cantilever beam configuration is given by ${ }^{22,23}$

$$
G=\frac{12\left(1-v^{2}\right) P^{2} a^{2}}{E t^{3} b^{2}}\left(1+0.64 \frac{t}{a}\right)^{2},
$$

where $a$ is the crack length, $E$ and $v$ are Young's modulus and Poisson's ratio of the substrate, $b$ is the width of the sample, and $t$ is the thickness of the substrate. The crack length $a$ can be evaluated from the sample compliance $S$ using ${ }^{22,23}$

$$
a=\left(\frac{E b S t^{3}}{8\left(1-v^{2}\right)}\right)^{1 / 3}-0.64 t
$$


where the compliance $S$ is defined as the crack opening distance, $h$, as measured at the open end of the sample, divided by the applied load $P$. Young's modulus and Poisson's ratio of the silicon substrate were taken as $168.9 \mathrm{GPa}$ and 0.064 , respectively, as appropriate for the crystallographic orientation of the silicon substrates. Using Eqns. (1) and (2), the energy release rate, i.e. the driving force for crack growth, and crack length can be evaluated at any given instance during the experiment.

The 4-PB test was carried out using the same load frame as for DCB test, but now equipped with a four-point bend fixture. A constant displacement rate of $0.3 \mu \mathrm{m} / \mathrm{s}$ was imposed at the loading pins, resulting in a crack growth rate of approximately $25 \mu \mathrm{m} / \mathrm{s}$. Most experimental curves displayed well-defined load plateaus during crack propagation, indicating a steady-state crack growth. The energy release rate under these conditions is independent of crack length, and is given by ${ }^{24}$

$$
G=\frac{21\left(1-v^{2}\right) P^{2} l^{2}}{16 E b^{2} t^{3}},
$$

where $l$ is the moment arm as determined by the position of the loading pins. The plateauvalue of the energy release rate is taken as the adhesion energy of the OSG/SiCN interface at this loading rate.

After the fracture tests, the fracture surfaces were examined by X-ray photoelectron spectroscopy (XPS) using an SSX-100 system (Surface Science Instrument Inc., Mountain View, CA) to determine the location of the crack path. The results confirmed that cracks propagate within the OSG layer for the DCB tests, and along the OSG/SiCN interface for the 4-PB adhesion tests.

We also investigated subcritical fracture of OSG coatings using the traditional approach of fracturing specimens in environments with controlled water partial pressure. In a typical subcritical DCB measurement, the baked-out specimen was first loaded at a constant displacement rate of $3 \mu \mathrm{m} / \mathrm{s}$. When $h$ is small and the pre-crack is stationary, the load increases linearly with $h$. Once $h$ reaches a threshold value, the pre-crack begins to grow and the load goes through a maximum. As soon as the load starts to decline, the crosshead is stopped. The evolution of the load is then recorded as the crack continues to grow under subcritical conditions, usually for several hours. From the load-displacement data, both crack velocity and the corresponding energy release rate can be calculated using Eqns. (1) and (2). We note that all samples were tested at a temperature of $21 \pm 1^{\circ} \mathrm{C}$. The relative humidity of the $\mathrm{N}_{2} / \mathrm{H}_{2} \mathrm{O}$ atmosphere in the environmental cell was kept at $11 \pm 0.5 \%, 49 \pm 0.5 \%, 66 \pm 0.5 \%$ and $87 \pm 0.5 \%$. 
Diffusion of water in the ULK-1 and ULK-1-pt film stacks was studied using an isotope tracer and dynamic secondary ion mass spectroscopy (SIMS). Working in depthprofiling mode, SIMS provides quantitative information of the composition profile through the film stack with nanometer level resolution. This is achieved by sputtering the surface of the specimen with a focused primary ion beam, producing secondary ions that are subsequently collected and analyzed by a mass spectrometer. For the SIMS measurement, ULK-1 and ULK-1-pt film stacks were first cleaved into $50 \mathrm{~mm} \times 30 \mathrm{~mm}$ stripes. The stripes were then baked at $190^{\circ} \mathrm{C}$ for 3 hours before soaking in a closed glass container at a temperature of $22 \pm 1{ }^{\circ} \mathrm{C}$ in a $10: 1$ by volume mixture of distilled water $\left(\mathrm{H}_{2}{ }^{16} \mathrm{O}\right)$ and isotope-labeled water $\left(\mathrm{H}_{2}{ }^{18} \mathrm{O}, 97 \%\right.$ concentrated, by Cambridge Isotope Laboratories, Inc., Andover, MA). The soaking time was 14.5 hour for ULK-1, and 15.8 hour for ULK-1-pt, respectively. The soaked samples were then superficially dried and loaded into the load lock of a SIMS system (Physical Electronics 6650 instrument; Chanhassen, MN). After 8 minutes of pumping, the samples were moved into the main chamber, where the stage holder had already been cooled with liquid nitrogen to minimize water diffusion during the measurement. Figure 1 illustrates the experimental configuration for the SIMS measurements schematically. Tracer concentration profiles were measured at several locations along the centerlines normal to the longer edges of the samples. A focused $5 \mathrm{kV} \mathrm{O}_{2}{ }^{+}$ion beam was used for sputtering, forming square craters with an edge length of approximately $0.2 \mathrm{~mm}$. Ions were collected from the central $15 \%$ of the crater areas and analyzed using a quadrupole mass spectrometer in the positive ion scan mode. The SIMS settings remained the same for all measurements. In addition to the ${ }^{18} \mathrm{O}$ signal, the ${ }^{12} \mathrm{C},{ }^{16} \mathrm{O}$ and ${ }^{28} \mathrm{Si}$ signals were also monitored. These signals were used to locate the $\mathrm{OSG} / \mathrm{SiCN}$ and $\mathrm{OSG} / \mathrm{Si}$ interface. The ${ }^{18} \mathrm{O}$ results reported in this paper were corrected for small intensity variation in the primary beam using the ${ }^{28} \mathrm{Si}$ signal as a reference. 


\section{Results and analysis}

\section{A. Adhesion degradation of the OSG/SiCN interface}

Figure 2 shows the adhesion energy of the $\mathrm{OSG} / \mathrm{SiCN}$ interface as a function of water immersion time for samples ULK-1 and ULK-1-liner. The error bars represent one standard deviation based on five or more measurements for each condition. Significant adhesion degradation is observed for both samples, indicating that water diffuses into the film stacks and causes a reduction of the interfacial adhesion energy as a result of a subcritical fracture process. The trend is similar to previous measurement for dense OSG coatings. ${ }^{12,13}$ Evidently, the degradation behavior is insensitive to the presence of the oxide film at the OSG/Si interface. A statistical analysis confirms with a 95\% confidence level that the difference between the two samples is not significant, indicating that the OSG/Si interface is unlikely to be the dominant diffusion path. We will show later that this conclusion is consistent with the SIMS measurements.

\section{B. Cohesive fracture of OSG}

Figure 3(a) shows a representative load $(P)$-displacement $(h)$ curve for a single DCB test on a ULK-1 sample after immersion in water for 7 hours. Once the crack propagates, the load decreases with increasing displacement. Several linear unloading curves are also shown in the figure. Figure 3(b) shows the corresponding energy release rate, $G$, as a function of displacement, calculated from Fig. 3(a) using Eqns. (1) and (2). It is evident from the figure that the crack propagates at a nearly constant value of the energy release rate, which we take as the cohesive fracture energy of the OSG. The theoretical $P-h$ relation that corresponds to this fracture energy is shown as the solid curve in Fig. 3(a). The good agreement with the experimental data is evident.

The cohesive fracture energies for all the OSG film stacks are summarized in Fig. 4. In contrast to the adhesion energy, the cohesive fracture energy remains nearly constant with water immersion time for all the OSG films. We will come back to explain this observation in the discussion section.

Figure 5 shows the subcritical cohesive fracture behavior of dry ULK-1 (squares) and ULK-1-liner (triangles) in environments with controlled water partial pressures. In the reaction-controlled regime, there is an exponential relationship between crack velocity and energy release rate. As the applied energy release rate decreases below a threshold value, crack growth stops. The value of the threshold energy release rate decreases with 
increasing relative humidity, indicating that the threshold is not a steric hindrance effect where water molecules are prevented from reaching the crack tip. Instead the threshold may be attributed to the existence of a corrosion threshold that depends on the environmental conditions. A similar trend has been observed for subcritical fracture in $\mathrm{SiO}_{2}$ film stacks. ${ }^{16}$ From the $v-G$ curves, it is evident that OSG is very susceptible to water-assisted stress corrosion: cracks grow faster if the relative humidity is increased at a given driving force; it requires less mechanical energy to drive the crack at a given velocity if more water molecules are present to facilitate bond severing. For a given level of relative humidity, there is little difference between the ULK-1 and ULK-1-liner samples, because water transported to the crack tip via the crack.

\section{Water-diffusion}

Depth profiling of the chemical composition was carried out for the ULK-1 and ULK-1-pt stacks exposed to ${ }^{18} \mathrm{O}$-labeled water for a given period of time, as well for control stacks that were not exposed to water. Figures 6(a) and 6(b) show the depth profile of ${ }^{18} \mathrm{O}$ for the ULK-1 before and after subtracting the control signal, respectively. The ${ }^{18} \mathrm{O}$ signal in the control sample is attributed to the natural abundance of ${ }^{18} \mathrm{O}$ in OSG; the intensity difference of ${ }^{18} \mathrm{O}$ between the soaked sample and the control sample is due to the ingress of labeled water. The net count of ${ }^{18} \mathrm{O}$ exhibits a sharp peak at the OSG/SiCN interface, and the intensity of the peak decreases with increasing distance to the sample edge. Figure 7(a) and 7(b) shows similar results for the ULK-1-pt sample. Again an ${ }^{18} \mathrm{O}$ peak is visible at the OSG/SiCN interface. The shape and overall trend of the peaks are similar to those for ULK-1, but at a given distance, the intensity of the peak is lower, indicating a lower water concentration. Note that the ${ }^{18} \mathrm{O}$ peak is unlikely to be a measurement artifact due to fast outgassing of water at the moment the SiCN capping layer is breached during the SIMS measurements because the samples were frozen during the measurements. Furthermore, the enhanced concentration of water at the OSG/SiCN interface is entirely consistent with the fracture experiments as will be discussed in the next section.

Figure 8 shows the normalized peak intensity of the ${ }^{18} \mathrm{O}$ signal as a function of diffusion distance for the ULK-1 and ULK-1-pt samples, respectively. The experimental data were normalized to have a unit surface concentration as determined by extrapolation with the complementary error function (solid curves in the figure). The water diffusion coefficient determined from the curve fits is $3.32 \pm 0.33 \times 10^{-11} \mathrm{~m}^{2} / \mathrm{s}$ for the ULK-1 sample and $3.37 \pm 0.18 \times 10^{-11} \mathrm{~m}^{2} / \mathrm{s}$ for the ULK-1-pt sample. For comparison, these 
values are approximately 6 orders of magnitude larger than for diffusion along the $\mathrm{SiO}_{2} / \mathrm{TiN}$ interface at a similar temperature, ${ }^{21}$ and they are 2-5 orders of magnitude larger than the diffusion coefficient of various aqueous solutions through porous MSSQ dielectric films of similar porosity. ${ }^{11}$

\section{Discussion}

The ${ }^{18} \mathrm{O}$ signals in the SIMS measurements exhibit sharp peaks at the OSG/SiCN interfaces of the soaked film stacks. The magnitude of the peaks decreases with increasing distance from the sample edge, evidence for a lateral composition gradient at the interface. The ${ }^{18} \mathrm{O}$ signal from the bulk of the OSG coatings, on the other hand, is uniformly weak and does not vary appreciably with distance to the sample edge. These observations indicate that water molecules tend to diffuse along the OSG/SiCN interface rather than through the bulk of the OSG.

This scenario is consistent with the fracture mechanics measurements, where the adhesion energy of the OSG/SiCN interface degrades significantly with increasing water immersion time, while the cohesive fracture energy of OSG remains largely unchanged. Evidently both sets of measurements support the rather surprising finding that diffusion occurs mainly along the OSG/SiCN interface and not through the porous OSG coating. We attribute the propensity of water to diffuse along the OSG/SiCN interface to the hydrophilic character of the interface. As-deposited OSG is hydrophobic. However, demethylation of the OSG surface during the initial phase of the $\mathrm{SiCN}$ deposition ${ }^{25}$ and/or during the interfacial He plasma treatment ${ }^{26}$ may lead to a hydrophilic interface by removing the organic component from the OSG.

To further examine this hypothesis, we use the observation by Guyer et al. ${ }^{11}$ that diffusion of pure water in a porous methyl silsesquioxane (MSSQ) dielectric is very slow, while diffusion of an aqueous solution of sodium hydroxide, sodium chloride, disodium ethylene diamine tetraacetate (EDTA), and glycine is fast. The argument is that the organic species in the solution act as surfactants rendering the surface of the OSG pores more hydrophilic and thus greatly enhancing the rate of water diffusion into the MSSQ films. Using the STT approach, we have measured the cohesive fracture energy of OSG after exposure to four different aqueous solutions containing the main components of the original solution from reference ${ }^{11}$ (solutions 1 through 4 in Table 2). Our DCB measurements show that the cohesive fracture energy of the ULK-1 OSG film degrades from $2.8 \pm 0.1 \mathrm{~J} / \mathrm{m}^{2}$ (dry sample) to $2.2 \pm 0.2 \mathrm{~J} / \mathrm{m}^{2}$ and $2.1 \pm 0.2 \mathrm{~J} / \mathrm{m}^{2}$, after 8.5 hours and 26.6 hours of submersion in solution 4 , a significantly greater reduction than for samples that were immersed in either distilled water (Fig. 4) or in the sodium hydroxide solution 
(\#1). Samples soaked in just the glycine solution (\#2) also showed a substantial reduction in cohesive fracture energy $\left(2.4 \pm 0.2 \mathrm{~J} / \mathrm{m}^{2}\right.$ after $6 \mathrm{hrs} ; 2.3 \pm 0.1 \mathrm{~J} / \mathrm{m}^{2}$ after $\left.25 \mathrm{hrs}\right)$, while samples soaked in the EDTA solution (\#3) did not. Water ingress for the pH-neutral glycine solution is slower than for the alkaline solution. This observation is in agreement with measurements by Guyer et al., ${ }^{11}$ who demonstrated that water diffusivity increases with $\mathrm{pH}$. This experiment demonstrates that the OSG cohesive fracture energy is degraded when water is present in the OSG and that glycine facilitates entry of water into OSG. This statement is further supported by contact angle measurements. Table 2 lists the contact angles of the solutions used in this study on both OSG and the fractured OSG/SiCN interface. For a given solution, the contact angle is always significantly smaller on the interface than on the OSG, in good agreement with our hypothesis that the interface is more hydrophilic than the OSG. Furthermore, solutions with glycine have smaller contact angles on both OSG and on the interface than solutions without glycine. Clearly, the hydrophobic character of the OSG and its interfaces plays an important role in determining whether water can enter the film stack and degrade its fracture properties.

It should finally be noted that the reduction in cohesive fracture energy of the OSG induced by solutions 2 and 4 is in line with the subcritical crack growth results shown in Fig. 5. The energy release rate to drive a crack at a velocity of $100 \mu \mathrm{m} / \mathrm{s}$ (i.e., the experimental velocity) indeed varies from 2.8 to approximately $2.0 \mathrm{~J} / \mathrm{m}^{2}$ depending on the water partial pressure. If OSG film stacks are exposed to water without any organic additives, on the other hand, water diffuses and accumulates along the interface and no reduction in cohesive fracture energy is observed.

The adhesion degradation result can be analyzed quantitatively using the model developed by Vlassak et al.. ${ }^{12,13}$ In this model, the water concentration profile along the crack front can be calculated from the diffusion time $t$. The concentration profile is then correlated with the overall energy release rate required to drive the crack at a given velocity using subcritical crack growth data. The adhesion energy as a function of $t$ is then given by

$$
G(t)=B-A\left[\ln \left(p_{v}\right)+\Phi(\gamma, \tau)\right]
$$

where $A$ and $B$ are material parameters that depend on crack velocity and that can be measured using a subcritical crack growth experiment,

$$
\Phi(\gamma, \tau)=\int_{0}^{1} \ln \left\{1-(1-\gamma)\left(\frac{4}{\pi} \sum_{k=0}^{\infty} \frac{1}{2 k+1} \sin ((2 k+1) \pi \xi) \exp \left(-(2 k+1)^{2} \pi^{2} \tau\right)\right)\right\} d \xi
$$

and 
$\gamma=\frac{p_{i}}{p_{v}}$, and $\tau=\frac{D_{\mathrm{H}_{2} \mathrm{O}}}{b^{2}} t$.

In these expressions, $p_{v}$ is the water vapor pressure, $p_{i}$ is the water partial pressure in equilibrium with the initial water content in the film stacks, $b$ is the width of the 4-PB specimens, and $D_{\mathrm{H}_{2} \mathrm{O}}$ is the diffusivity of water through the film stack. $D_{\mathrm{H}_{2} \mathrm{O}}$ and $\gamma$ are the only free variables in the model and they can be determined by fitting the experimental STT data. ${ }^{12,13}$ The solid curve in Fig. 2 is the best fit of Eq. (4) to the experimental data. The diffusion coefficient for the OSG/SiCN interface obtained from the fit is $9.8 \times 10^{-11} \mathrm{~m}^{2} / \mathrm{s}$, comparable to a value of $3.32 \times 10^{-11} \mathrm{~m}^{2} / \mathrm{s}$ obtained from the SIMS measurements. The dashed curve in Fig. 2 represents Eq. (4) evaluated using the SIMS diffusion coefficient - evidently the difference between both curves is quite small. For comparison, the diffusion coefficient of various aqueous solutions through a nanoporous MSSQ measured by Guyer et al. ${ }^{11}$ using an optical technique is approximately three orders of magnitude smaller than the diffusivity for the OSG/SiCN interface reported here.

Understanding water diffusion behavior in dielectric film stacks and its impact on the fracture properties of the materials and interfaces has important implications for the fabrication of interconnect structures. Wafers are repeatedly exposed to aqueous solutions during the interconnect fabrication process, allowing water to diffuse into the dielectric film stacks. Our investigation has shown that this is detrimental to the fracture properties of the film stack, and may result in delamination or cohesive failure. Evidently, the degree of hydrophobicity of the dielectric and any interface has an impact on how fast water can diffuse into the film stack. Controlling this property may provide a method to inhibit the ingress of water. Note, however, that organic components in the aqueous solutions can alter how water interacts with the dielectric and have a significant effect on the fracture behavior. Common organic additives such as isopropyl alcohol or acetic acid promote wetting of OSG and could have deleterious effects on the fracture behavior of OSG by allowing water to penetrate film stacks more easily. Note also that the water diffusivities for the film stacks used in this study are very large. The corresponding diffusion lengths on the time scale of the experiments are large - on the order of millimeters. Typical length scales in integrated circuits are at least three orders of magnitude smaller, so that the effect of water is important even if the diffusivity is six orders of magnitude smaller than the values reported in this study. Once water has penetrated the film stack, it is possible, however, to restore the fracture properties by annealing the samples - in a previous study, ${ }^{13}$ we showed that the degradation of the 
adhesive energy is entirely reversible and we would expect the same for the cohesive fracture energy.

\section{Conclusions}

In this paper, we have investigated the effect of water diffusion on the fracture behavior of nanoporous $\mathrm{OSG} / \mathrm{SiCN}$ film stacks. Isotope diffusion experiments show that water diffuses mainly along the OSG/SiCN interface and not through the porous films, thus causing significant degradation of the SiCN adhesion energy, while leaving the cohesive fracture energy of the OSG unchanged. This preference of water for the OSG/SiCN interface is attributed to the hydrophilic character of the interface. Indeed, degradation of the cohesive fracture energy is observed if the OSG film stack is exposed to aqueous solutions with organic additives that enhance wetting of the OSG. The adhesion degradation is well described by a simple diffusion/subcritical fracture model.

\section{Acknowledgements}

This work was supported by the National Science Foundation (NSF) under Grant CMS-0556169. The OSG film stacks were provided by Texas Instruments Incorporated. The authors acknowledge Dr. Tom Mates at the University of California, Santa Barbara for assistance with the SIMS measurements. 
Table 1. Specimen film stacks

\begin{tabular}{|l|l|c|c|}
\hline Sample index & Porosity & Relative dielectric constant of OSG & OSG/SiCN thickness (nm) \\
\hline ULK-1 (default) & $20.1 \%$ & 2.60 & $291.2 / 300$ \\
\hline ULK-1-pt* & $20.1 \%$ & 2.60 & $290.0 / 300$ \\
\hline ULK-1-liner** & $20.1 \%$ & 2.60 & $290 / 300$ \\
\hline ULK-2 & $44.5 \%$ & 2.30 & $239.4 / 300$ \\
\hline ULK-3 & $14.8 \%$ & 2.76 & $316.3 / 300$ \\
\hline
\end{tabular}

* OSG/SiCN was treated with a He plasma for 30 seconds.

**A 5-10 nm thick silicon dioxide layer was deposited between the Si and the OSG.

Table 2. Contact angle results for various solutions on the ULK-1 film and the OSG/SiNC interface measured using the static sessile drop method

\begin{tabular}{|c|c|c|c|c|c|c|c|}
\hline \multicolumn{3}{|c|}{ Solution Index } & $\# 1$ & $\# 2$ & $\# 3$ & $\# 4^{11}$ & $\# 5$ \\
\hline \multicolumn{3}{|l|}{ Solute } & $\mathrm{NaOH}$ & $\begin{array}{l}\text { glycine } \\
3.8 \mathrm{wt} \%\end{array}$ & $\begin{array}{l}\text { EDTA, } \\
0.0575 \mathrm{M}\end{array}$ & $\begin{array}{l}\mathrm{NaOH}, 2.1 \mathrm{wt} \% \\
\text { glycine, } 3.8 \mathrm{wt} \% \\
\text { EDTA, 1.1wt\% } \\
\mathrm{NaCl}, 3.0 \mathrm{wt} \%\end{array}$ & $\begin{array}{l}\text { Distilled } \\
\mathrm{H}_{2} \mathrm{O}\end{array}$ \\
\hline \multicolumn{3}{|l|}{$\mathrm{pH}$} & 11 & 5 & 4 & $11-12$ & $6-7$ \\
\hline \multirow{3}{*}{$\begin{array}{l}\text { Contact } \\
\text { angle }\end{array}$} & \multicolumn{2}{|c|}{ Fractured OSG surface } & $81 \pm 2^{\circ}$ & $72 \pm 1^{\circ}$ & $80 \pm 1^{\circ}$ & $76 \pm 1^{\circ}$ & $85 \pm 2^{\circ}$ \\
\hline & \multirow{2}{*}{$\begin{array}{l}\text { Delaminated } \\
\text { OSG/SiCN interface }\end{array}$} & OSG side & $50 \pm 1^{\circ}$ & $16 \pm 1^{\circ}$ & $38 \pm 1^{\circ}$ & $42 \pm 1^{\circ}$ & $71 \pm 1^{\circ}$ \\
\hline & & SiCN side & $42 \pm 1^{\circ}$ & $30 \pm 1^{\circ}$ & $29 \pm 1^{\circ}$ & $13 \pm 1^{\circ}$ & $80 \pm 1^{\circ}$ \\
\hline
\end{tabular}




\section{Figure caption list}

FIG.1. Schematic side view of the sample used for the SIMS measurements. The SiCN layer is an effective barrier to water diffusion, forcing water to diffuse into the film stack only from the edge.

FIG.2. Adhesion energy of the OSG/SiCN interface as a function of water immersion time for samples ULK-1 (square) and ULK-1-liner (circle). The solid curve is the best fit by the diffusion model ( $D=9.8 \pm 0.7$ $\mathrm{x} 10^{-11} \mathrm{~m}^{2} / \mathrm{s}$ ). The dotted curve is the prediction of the diffusion model using the diffusion coefficient obtained from the SIMS measurements $\left(\mathrm{D}=3.32 \times 10^{-11} \mathrm{~m}^{2} / \mathrm{s}\right)$.

FIG.3. DCB result for ULK-1 after immersion in water for 7 hours with (a) showing the original loaddisplacement data, and (b) the energy release rate as a function of load-point displacement. The solid curve in (a) shows the predictions by assuming a fracture resistance of $2.6 \mathrm{~J} / \mathrm{m}^{2}$, as marked in (b).

FIG.4. Cohesive fracture energy of various OSG films as a function of water immersion duration.

FIG.5. Crack velocity as a function of energy release rate for the cohesive fracture of OSG film stacks ULK-1 and ULK-1-liner.

FIG.6. ${ }^{18} \mathrm{O}$ concentration profile for ULK-1 (a) before and (b) after subtracting the signal obtained from the control sample.

FIG.7. ${ }^{18} \mathrm{O}$ concentration profile for ULK-1-pt (a) before and (b) after subtracting the signal obtained from the control sample.

FIG.8. Normalized peak intensity of the ${ }^{18} \mathrm{O}$ signal along the OSG/SiCN interface for ULK-1 and ULK-1-pt after subtracting the reference signal. The diffusion coefficient of water is calculated by fitting the experimental data with complementary error function. 


\section{References}

1 K. Maex, M. R. Baklanov, D. Shamiryan, F. Iacopi, S. H. Brongersma, and Z. S. Yanovitskaya, Journal of Applied Physics 93, 8793-8841 (2003).

M. Morgen, E. T. Ryan, J. H. Zhao, C. Hu, T. H. Cho, and P. S. Ho, Annual Review of Materials Science 30, 645-680 (2000).

A. Grill and D. A. Neumayer, Journal of Applied Physics 94, 6697-6707 (2003).

P. Y. Mabboux and K. K. Gleason, Journal of the Electrochemical Society 152, F7-F13 (2005).

Y. B. Lin, T. Y. Tsui, and J. J. Vlassak, Journal of the Electrochemical Society 153, F144-F152 (2006).

O. L. Mark, R. N. Vrtis, J. L. Vincent, A. S. Lukas, E. J. Karwacki, B. K. Peterson, and M. D. Bitner, in MRS Spring Meeting'2003:Symposium E Proceeding, San Francisco, USA, 2003 (Materials Research Society), p. E8.17

R. N. Vrtis, O. L. Mark, J. L. Vincent, A. S. Lukas, B. K. Peterson, M. D. Bitner, and E. J. Karwacki, in MRS Spring Meeting'2003:Symposium E Proceeding, San Francisco, USA, 2003 (Materials Research Society), p. E7.4

O. L. Mark, R. N. Vrtis, B. K. Peterson, M. K. Haas, S. J. Weigel, D. Wu, M. D. Bitner, and E. J. Karwacki, in MRS Spring Meeting'2006:Symposium F

Proceeding, San Francisco, USA, 2006 (Materials Research Society), p. 0914F01-02.

A. Grill, V. Patel, K. P. Rodbell, E. Huang, M. R. Baklanov, K. P. Mogilnikov, M. Toney, and H. C. Kim, Journal of Applied Physics 94, 3427-3435 (2003).

T. M. Shaw, D. Jimerson, D. Haders, C. E. Murray, A. Grill, D. C. Edelstein, and D. Chidambarrao, in Advanced Metallization Conference 2003 Proceeding (AMC'2003) Montreal, Quebec, Canada and Tokyo, Japan 2003 (Materials Research Society, Warrendale, PA), p. 77-84.

E. P. Guyer, J. Gantz, and R. H. Dauskardt, Journal of Materials Research 22, 710-718 (2007).

T. Y. Tsui, A. J. McKerrow, and J. J. Vlassak, Journal of the Mechanics and Physics of Solids 54, 887-903 (2006).

Y. B. Lin, T. Y. Tsui, and J. J. Vlassak, Acta Materialia 55, 2455-2464 (2007). J. J. Vlassak, Y. Lin, and T. Y. Tsui, Materials Science and Engineering AStructural Materials Properties Microstructure and Processing 391, 159-174 (2005).

E. P. Guyer, M. Patz, and R. H. Dauskardt, Journal of Materials Research 21, $882-$ 894 (2006).

M. W. Lane, X. H. Liu, and T. M. Shaw, IEEE Transactions on Device and Materials Reliability 4, 142-147 (2004).

S. M. Wiederhorn, Journal of the American Ceramic Society 50, 407 (1967).

S. M. Wiederhorn and L. H. Bolz, Journal of the American Ceramic Society 53, 543 (1970).

M. W. Lane, J. M. Snodgrass, and R. H. Dauskardt, Microelectronics Reliability 41, 1615-1624 (2001).

A. Grill, D. Edelstein, M. Lane, V. Patel, S. Gates, D. Restaino, and S. Molis, Journal of Applied Physics 103 (2008).

G. Xu, D. R. Clarke, Q. Ma, and H. Fujimoto, Journal of Applied Physics 88, 3695-3698 (2000). 
22 M. F. Kanninen, International Journal of Fracture 9, 83-92 (1973).

23 S. Li, J. Wang, and M. D. Thouless, Journal of the Mechanics and Physics of Solids 52, 193-214 (2004).

24 J. W. Hutchinson and Z. Suo, Advances in Applied Mechanics 29, 63-191 (1992).

25 T. Shaw, private communication (March 2, 2009).

26 J. Bao, H. Shi, J. Liu, H. Huang, P. S. Ho, M. D. Goodner, M. Moinpour, and G. M. Kloster, Journal of Vacuum Science \& Technology B 26, 219-226 (2008). 